\title{
LMI Criteria for Admissibility and Robust Stabilization of Singular Fractional-Order Systems Possessing Poly-Topic Uncertainties
}

\author{
Xuefeng Zhang *,+ ${ }^{*}$ and Jia Dong ${ }^{+}(\mathbb{D}$ \\ School of Sciences, Northeastern University, Shenyang 110819, China; 1800136@stu.neu.edu.cn \\ * Correspondence: zhangxuefeng@mail.neu.edu.cn \\ + These authors contributed equally to this work.
}

Received: 27 October 2020; Accepted: 10 December 2020; Published: 15 December 2020

\begin{abstract}
The issue of robust admissibility and control for singular fractional-order systems (FOSs) with polytopic uncertainties is investigated in this paper. Firstly, a new method based on linear matrix inequalities (LMIs) is presented to solve the admissibility problems of uncertain linear systems. Then, a solid criterion of robust admissibility and a corresponding state feedback controller are derived, which overcome the conservatism of the existing results. Finally, for the sake of demonstrating the validity of proposed results, some relevant examples are provided.
\end{abstract}

Keywords: fractional-order systems; stability criteria; admissibility; robust stabilization; linear matrix inequalities (LMIs)

\section{Introduction}

In recent years, the application of fractional calculus in the control field has gradually become a research hotspot. The importance of fractional-order systems (FOSs) increases day after day, and FOSs have obtained much attention [1-4]. Physical systems can be well described by using fractional differential equations, and fractional modeling can also be used in robotics, biomedicine, seismic analysis, and other practical fields. Lately, controllers [5-10] have been used to improve the robustness of closed-loop systems and enhance many systems' performance. In contrast to the classical integer-order controller, the fractional derivative has the memory property, so it can provide a powerful tool for modeling.

Stability analysis is the basis of FOSs, which is related to their order [11]. The existing LMI stability criteria are divided into two cases: the $(0,1)$ case and the $[1,2)$ case [12], respectively. In practical application, there are some uncertainties in many models, so these uncertainties are taken into account when modeling and performing analysis $[13,14]$. Uncertainties are considered in many systems; see the details in [15-20]. For example, for fractional-order linear time-invariant (FO-LTI) systems, a robust stability test method for FO-LTI systems with uncertain interval coefficients in the form of a state space was proposed in [17], which uses the method of determining the range of interval eigenvalues by matrix perturbation theory to overcome the difficulty of finding the argument of each eigenvalue. As an improvement of [17], a necessary and sufficient condition of testing the robust stability by using Lyapunov inequality to obtain a maximum eigenvalue of a Hermitian matrix was presented in [18]. A more exact robust stability condition for FOSs was proposed in [19]. Nonetheless, FO-LTI interval systems studied in [17-19] cannot accurately describe the perturbed FO-LTI systems. Therefore, the problem of the robust asymptotic stability of FOSs with structural perturbations was studied in [20], which is more general than the study of FOSs.

Recently, many basic concepts and results on the stability of normal integer-order systems have been successfully extended to singular integer-order systems and FOSs [21-25]. Sufficient conditions 
for the robust asymptotic stabilization of uncertain singular FOSs with $0<\alpha<2$ were proposed in [21]. However, the method is conservative, which involves the assumption that the system can be normalized. Therefore, this method is essentially a normal system solution method, rather than a singular system solution method. The difference and relation between the fractional-order system and integer-order system were pointed out in [22]. The stability properties of fractional-order differential system were presented in [11], which are the basis of some FOSs' stability analysis. The problem of robust stability and stabilization for fractional interval systems was studied in $[13,19]$. However, the uncertainties involved do not satisfy the norm bounded condition. Therefore, the stability and stabilization LMI criteria for fractional-order systems were developed based on D-stability [23], and new methods were introduced to deal with FOS control problems. The linear parameter-varying (LPV) discrete-time systems were studied in [24,25]. Under the assumption that all system state space matrices are uncertain parameters, the problem of robust state estimation was studied, and the necessary and sufficient conditions for the admissibility of discrete LPV systems were proposed in [24]. The design of a discrete time LPV system with $H_{\infty}$ gain-scheduled (GS) state feedback control was proposed for the first time to deal with the admissibility of LPV systems in [24].

Although it has made great contributions in both of the above aspects, up to now, for norm bounded uncertainties, the admissibility and robust stabilization of singular FOSs are still open problems. In the existing research, there are few stability analysis results for polytopic uncertainties. Necessary and sufficient conditions for robust stability with norm-bounded uncertainties were presented in [26]. It is noted that the sufficient condition for robust stability with polytopic-type uncertainties [26] is less conservative. Therefore, another brand-new model with polytopic uncertainties of FOSs was studied in [27]. The discussion of the order of systems considered including $(0,1)$ and $[1,2)$ was more comprehensive. Then, the sufficient condition for the robust stability of fractional-order uncertain linear systems was proposed. However, we find that Theorem 1 in [27] is questionable, and its conditions are conservative. Thus, in this paper, new criteria for the stability and robustness of polytopic uncertainties of FOSs are presented, and state feedback controllers are designed. In particular, the new method similar to [28] is used to deal with the admissibility issue of singular FOSs with the polytopic uncertainties, which improves the condition of Theorem 1 in [27] and overcomes the conservativeness. The proofs are also relatively simple. Then, some examples are given to elucidate the validity of the conclusions.

The organizational structure of this paper is as follows: In Section 2, a preliminary definition is introduced, and the model is established, then a related lemma is proposed. Some main results are provided in Section 3. Finally, four examples are given in Section 4 to testify to the significance of the approach. In Section 5, some conclusions are proposed to end the paper .

\section{Preliminaries}

Definition 1 ([4]). The Caputo fractional derivative of the function $x(t)$ is proposed as follows:

$$
D^{\alpha} x(t)=\frac{1}{\Gamma(n-\alpha)} \int_{0}^{t}(t-\tau)^{n-\alpha-1} x^{(n)}(\tau) d \tau,
$$

where the Euler Gamma function is represented as $\Gamma(\cdot)$ and $n$ is an integer that satisfies $n-1<\alpha \leq n$.

Lemma 1 ([28]). There are two conditions proposed as follows, which are equivalent:

1. Unforced System (1) with order $0<\alpha<1$ is admissible.

2. Some matrices $X, Y \in \mathbb{R}^{n \times n}, Q \in \mathbb{R}^{(n-m) \times n}$ exist such that Inequalities (2) and (3) hold or Inequalities (2) and (4) hold,

$$
E D^{\alpha} x(t)=A x(t)+B u(t)
$$




$$
\begin{gathered}
{\left[\begin{array}{cc}
X & Y \\
-Y & X
\end{array}\right]>0,} \\
\left\{A\left(a X E^{T}-b Y E^{T}+P Q\right)\right\}+\left\{A\left(a X E^{T}-b Y E^{T}+P Q\right)\right\}^{T}<0, \\
\left\{A^{T}(a X E-b Y E+\bar{P} Q)\right\}+\left\{A^{T}(a X E-b Y E+\bar{P} Q)\right\}^{T}<0,
\end{gathered}
$$

where $a$ and $b$ represent $\sin \left(\frac{\alpha \pi}{2}\right)$ and $\cos \left(\frac{\alpha \pi}{2}\right)$, respectively, and $P$ and $\bar{P}$ are given matrices, which have full column rank and satisfy $E P=0, E^{T} \bar{P}=0$.

\section{Problem Statement}

A mathematical model in ordinary physical systems is considered as follows [29]:

$$
\left(A_{n}+\Delta_{n}\right) E D^{n \alpha} \tilde{x}+\cdots+\left(A_{1}+\Delta_{1}\right) E D^{\alpha} \tilde{x}+\left(A_{0}+\Delta_{0}\right) \tilde{x}=F
$$

where $\alpha$ is the fractional commensurate order satisfying $0<\alpha<1 . A_{i} \in \mathbb{R}^{m \times m}$ are known nominal matrices, and $\Delta_{i} \in \mathbb{R}^{m \times m}$ are unknown. $F$ is a vector denoting a driving source, which is known. $D^{\alpha}(\tilde{x}), \cdots, D^{n \alpha}(\tilde{x})$ represent the differential of vectors $\tilde{x} . A_{n}+\Delta_{n}$ is invertible [30].

It is assumed that $z_{1}(t)=\tilde{x}(t), z_{2}(t)=E D^{\alpha} \tilde{x}(t), \cdots, z_{n}(t)=E D^{(n-1) \alpha} \tilde{x}(t)$. Then, we have the following formulas,

$$
\left\{\begin{array}{l}
E D^{\alpha} z_{1}(t)=z_{2}(t) \\
\vdots \\
E D^{\alpha} z_{n-1}(t)=z_{n}(t) \\
E D^{\alpha} z_{n}(t)=\left(A_{n}+\Delta_{n}\right)^{-1}\left[F-\left(A_{0}+\Delta_{0}\right) z_{1}(t) \cdots-\left(A_{n-1}+\Delta_{n-1}\right) z_{n}(t)\right]
\end{array}\right.
$$

The following formula can be derived as [29],

$$
-\left(A_{n}+\Delta_{n}\right)^{-1}\left(A_{i}+\Delta_{i}\right)=\left(I_{n}+A_{n}^{-1} \Delta_{n}\right)^{-1}\left(-A_{n}^{-1} A_{i}-A_{n}^{-1} \Delta_{i}\right) .
$$

According to Formulas (6) and (7), System (1) can be transformed into a new formula as follows,

$$
E D^{\alpha} x(t)=\left(I+\Delta_{I}\right)\left[\left(A+\Delta_{A}\right) x(t)+B u(t)\right],
$$

where $x(t) \in \mathbb{R}^{m n \times m n}$ is the pseudo-state vector and $A \in \mathbb{R}^{m n \times m n}$ and $B \in \mathbb{R}^{m n \times m}$ are the system parameter matrices. $E \in \mathbb{R}^{m n \times m n}$ is a singular matrix with $\operatorname{rank}(E)=r<m n$, and $D^{\alpha}$ is the fractional differential operator of order $\alpha$. In addition, $\Delta_{I}$ and $\Delta_{A}$ represent the uncertainties with the polytopic structure,

$$
\Delta_{I}=\sum_{i=1}^{P} r_{i} S_{i}, \Delta_{A}=\sum_{i=1}^{q} s_{i} F_{i}
$$

where both $r_{i}$ and $s_{i}$ represent uncertain parameters, and their range is $\left|r_{i}\right| \leq r$ and $\left|s_{i}\right| \leq s$, where both $r$ and $s$ are positive numbers and both $S_{i}$ and $F_{i}$ are given constant matrices that have appropriate dimensions. Therefore, according to Formula (9), the following inequalities can be obtained clearly,

$$
\Delta_{I} \Delta_{I}^{T} \leq H, \quad \Delta_{A} \Delta_{A}^{T} \leq G, \quad H=\sum_{i=1}^{P} p r^{2} S_{i} S_{i}^{T}, \quad G=\sum_{i=1}^{q} q s^{2} F_{i} F_{i}^{T} .
$$


System (8) is globally stabilizable where a controller $u(t)=K x(t)$ is designed,

$$
E D^{\alpha} x(t)=\left(A+B K+\Delta_{A}+\Delta_{I} A+\Delta_{I} B K+\Delta_{I} \Delta_{A}\right) x(t) .
$$

If $u(t)=0$, System (8) is reduced to the following form, whose uncertain parameters are second-order nonlinear,

$$
E D^{\alpha} x(t)=\left(A+\Delta_{A}+\Delta_{I} A+\Delta_{I} \Delta_{A}\right) x(t),
$$

where $\Delta_{I} \Delta_{A}$ denotes that the second-order uncertain parameter is unknown.

Remark 1. It is easy to find that the FO-LTI interval uncertain systems studied in [17-20] are described by the system:

$$
D^{\alpha} x(t)=\left(A+\Delta_{A}\right) x(t)+B u(t),
$$

and these results may not be applicable to FOS (8). For FO-LTI systems with second-order nonlinear uncertain parameters, there are few research results on the robust stability, and the parameters' uncertainties considered in [20] are provided in linear form rather than nonlinear form as System (8).

\section{Main Results}

In this section, some new criteria for the robust admissibility problems of uncertain FOSs are proposed.

Theorem 1. Unforced System (8) is admissible if some matrices $X, Y \in \mathbb{R}^{m n \times m n}, Q_{1} \in \mathbb{R}^{(m n-r) \times m n}$, and some scalars $\varepsilon_{1}>0$ and $\eta_{1}>0$ satisfy Formula (2) and:

$$
\left[\begin{array}{cc}
\Omega_{11} & \Omega_{12} \\
\star & \Omega_{22}
\end{array}\right]<0,
$$

where:

$$
\begin{gathered}
\Omega_{11}=\operatorname{sym}\left\{A\left(a X E^{T}-b Y E^{T}+P Q_{1}\right)\right\}+\varepsilon_{1} G+H \\
\Omega_{12}=\left[\left(a X E^{T}-b Y E^{T}+P Q_{1}\right)^{T}\left(a X E^{T}-b Y E^{T}+P Q_{1}\right)^{T} A^{T} \quad\left(a X E^{T}-b Y E^{T}+P Q_{1}\right)^{T}\right], \\
\Omega_{22}=-\operatorname{diag}\left(\varepsilon_{1} I\left(I-\eta_{1} G\right) I \quad \eta_{1} I\right) .
\end{gathered}
$$

Proof. Unforced System (8) is obtained in the following form,

$$
E D^{\alpha} x(t)=\widetilde{A} x(t)
$$

where $\widetilde{A}=A+\Delta_{I} A+\Delta_{A}+\Delta_{I} \Delta_{A}$.

In light of Lemma 1, we can obtain that Equation (14) is admissible if some matrices $X, Y \in$ $\mathbb{R}^{m n \times m n}, Q_{1} \in \mathbb{R}^{(m n-r) \times m n}$ satisfy Formula (2) and:

$$
\operatorname{sym}\left\{\widetilde{A}\left(a X E^{T}-b Y E^{T}+P Q_{1}\right)\right\}=\operatorname{sym}\left\{\left(A+\Delta_{I} A+\Delta_{A}+\Delta_{I} \Delta_{A}\right)\left(a X E^{T}-b Y E^{T}+P Q_{1}\right)\right\}<0 .
$$

In order to facilitate the analysis, Formula (15) mentioned above is expanded as follows,

$$
\begin{gathered}
\operatorname{sym}\left\{A\left(a X E^{T}-b Y E^{T}+P Q_{1}\right)+\Delta_{A}\left(a X E^{T}-b Y E^{T}+P Q_{1}\right)\right. \\
\left.+\Delta_{I}\left(A+\Delta_{A}\right)\left(a X E^{T}-b Y E^{T}+P Q_{1}\right)\right\}<0 .
\end{gathered}
$$

For arbitrary $\varepsilon>0$, we have the following inequality,

$$
\left(\varepsilon^{\frac{1}{2}} X-\varepsilon^{-\frac{1}{2}} Y\right)^{T}\left(\varepsilon^{\frac{1}{2}} X-\varepsilon^{-\frac{1}{2}} Y\right)=\varepsilon X^{T} X+\varepsilon^{-1} Y^{T} Y-Y^{T} X-X^{T} Y \geq 0,
$$


then we can conclude that,

$$
X^{T} Y+Y^{T} X \leq \varepsilon X^{T} X+\varepsilon^{-1} Y^{T} Y
$$

According to Inequality (18) and Lemma 2 in [31], there exist two scalars $\varepsilon_{i}, i=1,2$ such that:

$$
\begin{gathered}
\operatorname{sym}\left(\Delta_{A}\left(a X E^{T}-b Y E^{T}+P Q_{1}\right)\right) \\
\leq \varepsilon_{1} \Delta_{A} \Delta_{A}^{T}+\varepsilon_{1}^{-1}\left(a X E^{T}-b Y E^{T}+P Q_{1}\right)^{T}\left(a X E^{T}-b Y E^{T}+P Q_{1}\right) \\
\leq \varepsilon_{1} G+\varepsilon_{1}^{-1}\left(a X E^{T}-b Y E^{T}+P Q_{1}\right)^{T}\left(a X E^{T}-b Y E^{T}+P Q_{1}\right) .
\end{gathered}
$$

Similar to Formula (19), we have:

$$
\begin{gathered}
\operatorname{sym}\left\{\Delta_{I}\left(A+\Delta_{A}\right)\left(a X E^{T}-b Y E^{T}+P Q_{1}\right)\right\} \\
\leq \varepsilon_{2} H+\varepsilon_{2}\left(a X E^{T}-b Y E^{T}+P Q_{1}\right)^{T}\left(A+\Delta_{A}\right)^{T}\left(A+\Delta_{A}\right)\left(a X E^{T}-b Y E^{T}+P Q_{1}\right) .
\end{gathered}
$$

For arbitrary $\eta_{1}>0$ satisfying $I-\eta_{1} G>0$, from Inequality (10), it is obtained that,

$$
I-\eta_{1} \Delta_{A} \Delta_{A}^{T}>I-\varepsilon_{1} G>0,
$$

which means that:

$$
\left(I-\eta_{1} \Delta_{A} \Delta_{A}^{T}\right)^{-1}<\left(I-\eta_{1} G\right)^{-1} .
$$

From Lemma 2 in [31], it follows that:

$$
\left(A+\Delta_{A}\right)^{T}\left(A+\Delta_{A}\right) \leq A^{T}\left(I-\eta_{1} \Delta_{A} \Delta_{A}^{T}\right)^{-1} A+\eta_{1}^{-1} I<A^{T}\left(I-\eta_{1} G\right)^{-1} A+\eta_{1}^{-1} I .
$$

According to Inequalities (20) and (21), for simplicity, setting $\varepsilon_{2}=1$, we have the following inequality,

$$
\begin{gathered}
\operatorname{sym}\left\{\Delta_{I}\left(A+\Delta_{A}\right)\left(a X E^{T}-b Y E^{T}+P Q_{1}\right)\right\} \\
<H+\left(a X E^{T}-b Y E^{T}+P Q_{1}\right)^{T}\left\{A^{T}\left(I-\eta_{1} G\right)^{-1} A+\eta_{1}^{-1} I\right\}\left(a X E^{T}-b Y E^{T}+P Q_{1}\right) .
\end{gathered}
$$

Substituting Inequalities (19) and (22) into Formula (16) yields:

$$
\begin{gathered}
\operatorname{sym}\left\{\widetilde{A}\left(a X E^{T}-b Y E^{T}+P Q_{1}\right)\right\}<\operatorname{sym}\left\{A\left(a X E^{T}-b Y E^{T}+P Q_{1}\right)\right\} \\
+\varepsilon_{1} G+\varepsilon_{1}^{-1}\left(a X E^{T}-b Y E^{T}+P Q_{1}\right)^{T}\left(a X E^{T}-b Y E^{T}+P Q_{1}\right) \\
+H+\left(a X E^{T}-b Y E^{T}+P Q_{1}\right)^{T}\left\{A^{T}\left(I-\eta_{1} G\right)^{-1} A+\eta_{1}^{-1} I\right\}\left(a X E^{T}-b Y E^{T}+P Q_{1}\right)<0 .
\end{gathered}
$$

At last, the relationship is equivalent between Formulas (23) and (13).

Theorem 2. If matrices $X, Y \in \mathbb{R}^{m n \times m n}, Q_{2} \in \mathbb{R}^{(m n-r) \times m n}$, and some scalars $\gamma_{1}, \gamma_{3}$, and $\eta_{2}>0$ satisfy Inequality (9) and:

$$
\left[\begin{array}{cc}
\Gamma_{11} & \Gamma_{12} \\
\star & \Gamma_{22}
\end{array}\right]<0
$$

where:

$$
\begin{gathered}
\Gamma_{11}=\operatorname{sym}\left\{A\left(a X E^{T}-b Y E^{T}+P Q_{2}\right)+B Z\right\}+\gamma_{1} G+H+\gamma_{3} H, \\
\Gamma_{12}=\left[\begin{array}{cccc}
\left(a X E^{T}-b Y E^{T}+P Q_{2}\right)^{T} & \left(a X E^{T}-b Y E^{T}+P Q_{2}\right)^{T} A^{T} & \left(a X E^{T}-b Y E^{T}+P Q_{2}\right)^{T} & Z^{T} B^{T}
\end{array}\right], \\
\Gamma_{22}=-\operatorname{diag}\left(\begin{array}{llll}
\gamma_{1} I & \left(I-\eta_{2} G\right) I & \eta_{2} I & \left.\gamma_{3} I\right) .
\end{array}\right.
\end{gathered}
$$

Unforced System (8) is admissible. In addition, a controller is obtained by $K=Z\left(a X E^{T}-b Y E^{T}+P Q_{2}\right)^{-1}$. 
Proof. Consider $\bar{A}=A+B K$, then $\widehat{A}=\bar{A}+\Delta_{I} \bar{A}+\Delta_{A}+\Delta_{I} \Delta_{A}$, and on the basis of Lemma 1, we can obtain that System (8) is admissible if two matrices $X, Y \in \mathbb{R}^{m n \times m n}$ satisfy Inequality (2) and:

$$
\begin{gathered}
\operatorname{sym}\left\{\widehat{A}\left(a X E^{T}-b Y E^{T}+P Q_{2}\right)\right\} \\
=\operatorname{sym}\left\{(A+B K)\left(a X E^{T}-b Y E^{T}+P Q_{2}\right)+\Delta_{A}\left(a X E^{T}-b Y E^{T}+P Q_{2}\right)\right. \\
\left.+\Delta_{I}\left(A+\Delta_{A}\right)\left(a X E^{T}-b Y E^{T}+P Q_{2}\right)+\Delta_{I} B K\left(a X E^{T}-b Y E^{T}+P Q_{2}\right)\right\}<0 .
\end{gathered}
$$

Similar to the proof of Theorem 1, it follows from Inequality (18) and Lemma 2 in [31] that there exist some scalars $\gamma_{i}, i=1,2,3$, and $\eta_{2}>0$ such that:

$$
\begin{gathered}
\operatorname{sym}\left\{\Delta_{A}\left(a X E^{T}-b Y E^{T}+P Q_{2}\right)\right\} \\
\left.\leq \gamma_{1} G+\gamma_{1}^{-1}\left(a X E^{T}-b Y E^{T}+P Q_{2}\right)^{T}\left(a X E^{T}-b Y E^{T}+P Q_{2}\right)\right) .
\end{gathered}
$$

For simplicity, set $\gamma_{2}=1$. The following inequality is the same as Inequality (26),

$$
\begin{gathered}
\operatorname{sym}\left\{\Delta_{I}\left(A+\Delta_{A}\right)\left(a X E^{T}-b Y E^{T}+P Q_{2}\right)\right\} \\
<H+\left(a X E^{T}-b Y E^{T}+P Q_{2}\right)^{T}\left\{A^{T}\left(I-\eta_{2} G\right)^{-1} A+\eta_{2}^{-1} I\right\}\left(a X E^{T}-b Y E^{T}+P Q_{2}\right),
\end{gathered}
$$

Letting $Z=K\left(a X E^{T}-b Y E^{T}+P Q_{2}\right)$, it yields

$$
\operatorname{sym}\left(\Delta_{I} B K\left(a X E^{T}-b Y E^{T}+P Q_{2}\right)\right)=\Delta_{I} B Z+Z^{T} B^{T} \Delta_{I}^{T} \leq \gamma_{3} H+\gamma_{3}^{-1} Z^{T} B^{T} B Z .
$$

Then, substituting Inequalities (26), (27), and (28) into Formula (25) yields:

$$
\begin{gathered}
\operatorname{sym}\left\{\widehat{A}\left(a X E^{T}-b Y E^{T}+P Q_{2}\right)\right\} \\
<\operatorname{sym}\left\{(A+B K)\left(a X E^{T}-b Y E^{T}+P Q_{2}\right)\right\}+\gamma_{1} G \\
+\gamma_{1}^{-1}\left(a X E^{T}-b Y E^{T}+P Q_{2}\right)^{T}\left(a X E^{T}-b Y E^{T}+P Q_{2}\right) \\
+H+\left(a X E^{T}-b Y E^{T}+P Q_{2}\right)^{T}\left\{A\left(I-\eta_{2} G\right)^{-1} A+\eta_{2} I\right\}\left(a X E^{T}-b Y E^{T}+P Q_{2}\right) \\
+\gamma_{3} H+\gamma_{3}^{-1} Z^{T} B^{T} B Z<0 .
\end{gathered}
$$

At the end of this proof, using the Schur complement in [32], the relationship between Inequalities (29) and (26) is equivalent.

Remark 2. The comparisons in $[12,14,27]$ are provided in Table 1, and we find that their theorems involve many real decision variables $\left(P_{11}, P_{12}, P_{21}, P_{22}\right)$, under the strict assumptions of $P_{11}=P_{21}>0$ and $P_{12}=P_{22}=0$ in [14], which limit the extension to the controller design. The results are conservative.

Table 1. Comparisons with existing results.

\begin{tabular}{ccccc}
\hline Reference & Variable Kind & Variables & Easy to Solve? & Less Conservative? \\
\hline$[2]$ & $\mathrm{R}$ & 1 & yes & no \\
\hline$[12]$ & $\mathrm{C}$ & 4 & no & no \\
\hline$[14]$ & $\mathrm{R}$ & 4 & no & no \\
\hline$[27]$ & $\mathrm{R}$ & 2 & no & no \\
\hline ours & $\mathrm{R}$ & 2 & yes & yes \\
\hline
\end{tabular}

\section{Numerical Examples}

In this section, some examples are presented to illustrate the effectiveness of our theoretical results.

Example 1. In this case, consider an electrical circuit drawn in Figure 1 with given resistances $R_{i}, i=1,2,3$, inductances $L_{i}, i=1,2,3$, and source voltages $e_{1}$ and $e_{2}$ [33]. 
Then, we obtain the equations as follows by using Kirchhoff's laws:

$$
\begin{gathered}
e_{1}=R_{1} i_{1}+L_{1} D^{\alpha} i_{1}+R_{3} i_{3}+L_{3} D^{\alpha} i_{3}, \\
e_{2}=R_{2} i_{2}+L_{2} D^{\alpha} i_{2}+R_{3} i_{3}+L_{3} D^{\alpha} i_{3}, \\
i_{3}=i_{1}+i_{2},
\end{gathered}
$$

then the following system is obtained,

$$
E D^{\alpha} x(t)=A x(t)+B u(t),
$$

where:

$$
\begin{gathered}
\alpha=0.2, \quad x(t)=\left[\begin{array}{lll}
i_{1}^{T} & i_{2}{ }^{T} & i_{3}{ }^{T}
\end{array}\right]^{T}, u(t)=\left[\begin{array}{ll}
e_{1}{ }^{T} & e_{2}{ }^{T}
\end{array}\right]^{T}, \\
E=\left[\begin{array}{ccc}
L_{1} & 0 & L_{3} \\
0 & L_{2} & L_{3} \\
0 & 0 & 0
\end{array}\right], A=\left[\begin{array}{ccc}
-R_{1} & 0 & -R_{3} \\
0 & -R_{2} & -R_{3} \\
1 & 1 & -1
\end{array}\right], B=\left[\begin{array}{ll}
1 & 0 \\
0 & 1 \\
0 & 0
\end{array}\right] .
\end{gathered}
$$

For simplicity, let $\Delta_{A}=\Delta_{I}=0$, and System (29) is exactly FOS (1). Set:

$$
L_{i}=1, i=1,2,3, R_{1}=1, R_{2}=3, R_{3}=2 \text {. }
$$

Using the MATLAB LMI toolbox, we obtain that (24) is feasible, which illustrates that System (30) is admissible, and the state feedback gain is obtained as follows,

$$
K=\left[\begin{array}{ccc}
-3.0041 & -2.0744 & 0.9194 \\
0.6682 & -0.6888 & -1.3623
\end{array}\right] \text {. }
$$

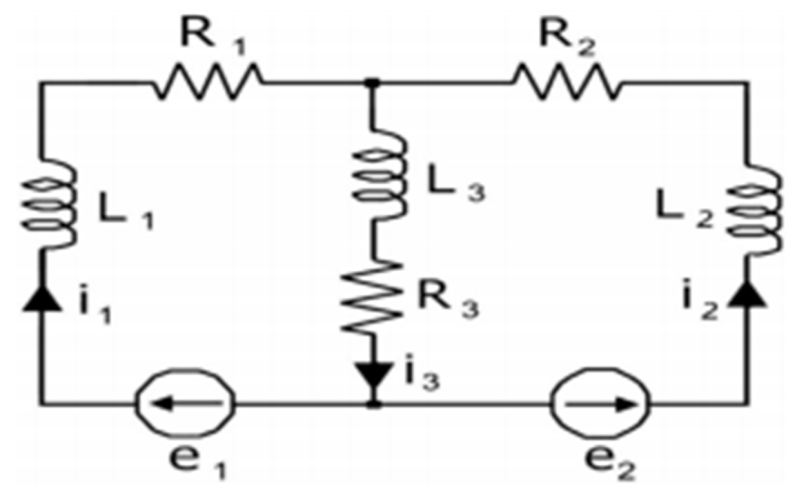

Figure 1. Electronic network

Example 2. In this case, consider unforced System (8) with $\alpha=0.5$ and:

$$
\begin{gathered}
A=\left[\begin{array}{lll}
-2 & -1 & -1 \\
-2 & -3 & -1 \\
-1 & -1 & -4
\end{array}\right], H=\left[\begin{array}{ccc}
0.12 & 0 & 0 \\
0 & 0 & 0 \\
0 & 0 & 0.12
\end{array}\right], \\
G=\left[\begin{array}{ccc}
0 & 0 & 0 \\
0 & 0.16 & 0.16 \\
0 & 0.16 & 0.16
\end{array}\right], P=\left[\begin{array}{c}
-1 \\
1 \\
-1
\end{array}\right] .
\end{gathered}
$$


$|\arg (\operatorname{spec}(A))|>\frac{\alpha \pi}{2}$. The feasible solutions of Inequalities (2) and (13) in Theorem 1 are obtained as follows:

$$
\begin{gathered}
\varepsilon_{1}=0.9214, \eta_{1}=0.9447 \\
X=\left[\begin{array}{ccc}
0.4872 & -0.3191 & 0.1680 \\
-0.3191 & 0.3740 & -0.2812 \\
0.1680 & -0.2812 & 0.5251
\end{array}\right], Y=\left[\begin{array}{ccc}
0 & -0.0443 & -0.0443 \\
0.0443 & 0 & -0.0443 \\
0.0443 & 0.0443 & 0
\end{array}\right], \\
Q_{1}=\left[\begin{array}{lll}
-0.0767 & 0.1154 & -0.0835
\end{array}\right] .
\end{gathered}
$$

In light of Theorem 1, we conclude that unforced System (8) is admissible.

Example 3. Consider System (8) with parameters as $\alpha=0.5$ and:

$$
A=\left[\begin{array}{ccc}
-2 & 0 & 1 \\
-2 & -4 & 0 \\
-1 & -2 & -3
\end{array}\right], B=\left[\begin{array}{l}
0.15 \\
0.35 \\
0.55
\end{array}\right]
$$

Using the MATLAB LMI toolbox, we obtain the feasible solutions of Inequalities (2) and (24) in Theorem 2 as follows:

$$
\begin{gathered}
\gamma_{1}=0.9261 \gamma_{3}=0.9280 \eta_{2}=0.9343 \\
X=\left[\begin{array}{ccc}
0.4389 & -0.3044 & 0.2188 \\
-0.3044 & 0.3453 & -0.3124 \\
0.2188 & -0.3124 & 0.4309
\end{array}\right], Y=\left[\begin{array}{ccc}
0 & -0.0341 & -0.0341 \\
0.0341 & 0 & -0.0341 \\
0.0341 & 0.0341 & 0
\end{array}\right], \\
Z=\left[\begin{array}{lll}
-0.0257 & -0.1704 & -0.5066
\end{array}\right], Q_{2}=\left[\begin{array}{lll}
0.0579 & -0.0713 & 0.0736
\end{array}\right],
\end{gathered}
$$

and the state feedback gain is obtained as follows:

$$
K=Z\left(a X E^{T}-b Y E^{T}+P Q_{2}\right)^{-1}=\left[\begin{array}{lll}
-4.1971 & -5.1599 & -7.8445
\end{array}\right] .
$$

Thus, System (8) is asymptotically admissible. The transients of the state variables are shown in Figure 2, which confirms the admissibility of System (8).

Example 4. Consider a polytopic-type uncertain FOS (6) in [27] with the following parameters:

$$
\alpha=0.5, \quad A=\left[\begin{array}{ccc}
6 & -3 & 0 \\
3 & 6 & 0 \\
0 & 0 & -5
\end{array}\right], H=\left[\begin{array}{ccc}
0.018 & 0 & 0 \\
0 & 0 & 0 \\
0 & 0 & 0.018
\end{array}\right], G=\left[\begin{array}{ccc}
0 & 0 & 0 \\
0 & 0.036 & 0 \\
0 & 0 & 0.036
\end{array}\right]
$$

The three eigenvalues of matrix $A$ are -5 and $6 \pm 3 j$, respectively, which do not meet the condition of $|\arg (\operatorname{spec}(A))|>\frac{\alpha \pi}{2}$. Using the MATLAB LMI toolbox, we can obtain the best value of $t$ from Inequalities (2) and (13) in Theorem 1. In this present paper, $t=0.0031>0$, which means that these LMI constraints were found to be infeasible. From Lemma 1, it follows that System (6) in [27] is unstable even if $\Delta_{I}=\Delta_{A}=0$. However, the LMI of (17) in [27] for this example has feasible solutions:

$$
\begin{gathered}
P_{11}=P_{21}=\left[\begin{array}{ccc}
0.0036 & 0.0002 & -0.0003 \\
0.0002 & 0.0010 & -0.0040 \\
-0.0003 & -0.0040 & 0.1466
\end{array}\right], P_{12}=-P_{22}=\left[\begin{array}{ccc}
0 & 00.0393 & -0.0014 \\
0.0393 & 0 & -0.0054 \\
0.0014 & 0.0054 & 0
\end{array}\right], \\
\varepsilon_{1}=5.3572, \quad \varepsilon_{11}=\varepsilon_{21}=1.0359, \quad \varepsilon_{12}=\varepsilon_{22}=1.662 .
\end{gathered}
$$


According to Theorem 1 in [27], we can conclude that System (6) in [27] is stable, which is thewrong conclusion.

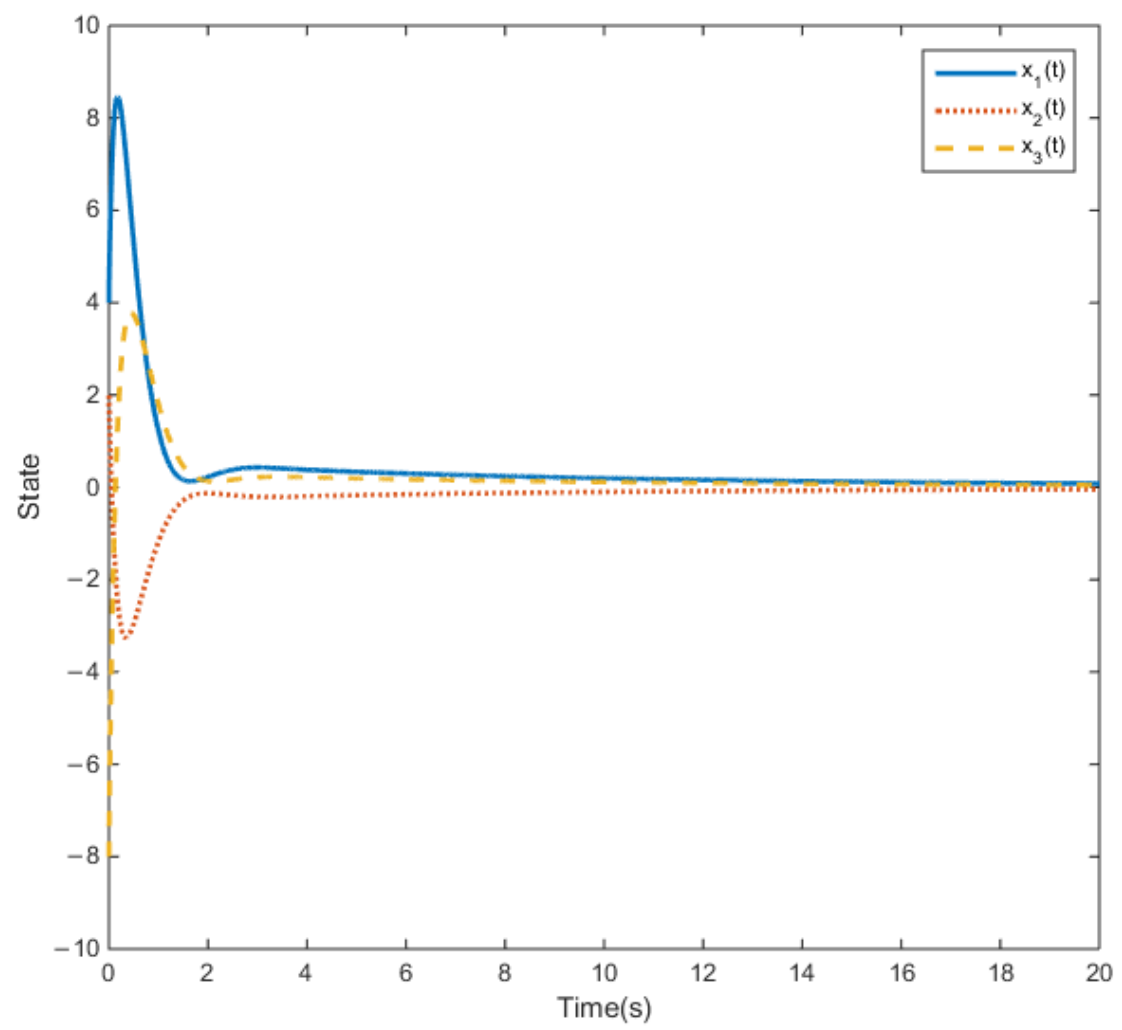

Figure 2. State response curves of System (9) in Example 3.

\section{Conclusions}

In this paper, the robust admissibility of FOSs with polytopic uncertainties is studied. Sufficient conditions for the robust admissibility of FOSs are proposed based on a new LMI method. Then, the corresponding criteria for the robust stabilization of FOSs with polytopic uncertainties are presented by designing feedback controllers. Finally, some examples are proposed to verify the results. The admissibility of singular FOSs with uncertain description matrix $E$ will be studied in the future work.

Author Contributions: Conceptualization, X.Z.; Data curation, J.D.; Formal analysis, X.Z. and J.D.; Investigation, X.Z.; Methodology, X.Z.; Software, J.D.; Writing original draft, J.D.; Writing review and editing, X.Z. All authors have read and agreed to the published version of the manuscript.

Funding: This research received no external funding

Conflicts of Interest: The authors declare no conflict of interest.

\section{References}

1. Podlubny, I. Fractional Differential Equations; Academic Press: San Dieage, CA, USA, 1999.

2. Aguila-Gamacho, N.; Duarte-Mermound, M.A.; Gallegos, J.A. Lyapunov Functions for Fractional Order Systems. Commun. Nonlinear Sci. Numer. Simulat. 2014, 19, 2951-2957. [CrossRef]

3. Zhang, X.F. Relationship between integer-order systems and fractional-order system and its two applications. IEEE/CAA J. Autom. Sin. 2018, 5, 639-643. [CrossRef]

4. Zhang, R.M.; Liu, X.Z.; Zeng, D.Q.; Zhong, S.M.; Shi, K.B. A Novel Approach to Stability and Stabilization of Fuzzy Sampled-data Markovian Chaotic Systems. Fuzzy Sets Syst. 2018, 344, 108-128. [CrossRef]

5. Chen, J.; Zhuang, B.; Chen, Y.Q.; Cui, B.T. Diffusion Control for A Tempered Anomalous Diffusion System Using Fractional-Order PI Controllers. ISA Trans. 2018, 82, 94-106. [CrossRef] 
6. Zhang, J.X.; Yang, G.H. Fault-tolerant output-constrained control of unknown Euler-Lagrange systems with prescribed tracking accuracy. Automatica 2020, 111, 108606. [CrossRef]

7. Zhang, J.X.; Yang, G.H. Low-complexity tracking control of strict-feedback systems with unknown control directions. IEEE Trans. Autom. Control 2019, 64, 5175-5182. [CrossRef]

8. Dastjerdi, A.A.; Vinagre, B.M.; Chen, Y.Q.; HosseinNia, S.H. Linear Fractional Order Controllers; A Survey in The Frequency Domain. Annu. Rev. Control 2019, 47, 51-70. [CrossRef]

9. Talegon, D.F.; Batlle, V.F.; Tejado, I.; Vinagre, B.M.; HosseinNia, S.H. Stable Force Control And Contact Transition of A Single Link Flexible Robot Using A Fractional-Order Controller. ISA Trans. 2019, 89, 139-157. [CrossRef]

10. Zhang, J.X.; Yang, G.H. Prescribed performance fault-tolerant control of uncertain nonlinear systems with unknown control directions. IEEE Trans. Autom. Control 2017, 62, 6529-6535. [CrossRef]

11. Matignon, D. Stability Properties for Generalized Fractional Differential Systems. Proc. ESAIM 1998, 5, 145-158. [CrossRef]

12. Sabatier, J.; Moze, M.; Farges, C. LMI Stability Conditions for Fractional-Order Systems. Comput. Math. Appl. 2010, 59, 1594-1609. [CrossRef]

13. Lu, J.G.; Chen, G.R. Robust Stability and Stabilization of Fractional-Order Interval Systems: An LMI Approach. IEEE Trans. Autom. Control 2009, 54, 1294-1299.

14. Lu, J.G.; Chen, Y.Q. Robust Stability and Stabilization of Fractional-Order Interval Systems with the Fractional Order $\alpha$ : The $0<\alpha<1$ Case. IEEE Trans. Autom. Control 2010, 55, 152-158.

15. Wei, Y.H.; Tse, P.W.; Yao, Z.; Wang, Y. The Output Feedback Control Synthesis for A Class of Singular Fractional Order Systems. ISA Trans. 2017, 69, 1-9. [CrossRef]

16. Kai, B.S.; Wang, J.; Zhong, S.M.; Zhang, X.J.; Liu, Y.J.; Cheng, J. New Reliable Nonuniform Sampling Control for Uncertain Chaotic Neural Networks Under Markov Switching Topologies. Appl. Math. Comput. 2019, 347, 169-193.

17. Chen, Y.Q.; Ahn, H.S.; Podlubny, I. Robust Stability Check of Fractional Order Linear Time Invariant Systems with Interval Uncertainties. Signal Process. 2006, 86, 2611-2618. [CrossRef]

18. Ahn, H.S.; Chen, Y.Q.; Podlubny, I. Robust Stability Test of A Class of Linear Time- Invariant Interval Fractional-Order System Using Lyapunov Inequality. Comput. Math. Appl. 2007, 187, 27-34. [CrossRef]

19. Ahn, H.S.; Chen, Y.Q. Necessary And Sufficient Stability Condition of Fractional-Order Interval Linear Systems. Automatica 2008, 44, 2985-2988. [CrossRef]

20. Lu, J.G.; Chen, Y.Q.; Chen, W.D. Robust Asymptotical Stability of Fractional-Order Linear Systems with Structured Perturbations. Comput. Math. Appl. 2013, 66, 873-882. [CrossRef]

21. Doyea, I.N.; Darouachb, M.; Zasadzinski, M.; Radhy, N.-E. Robust Stabilization of Uncertain Descriptor Fractional-Order Systems. Automatica 2013, 49, 1907-1913.

22. Zhang, X.F.; Chen, Y.Q. Remarks on Fractional Order Control Systems. In Proceedings of the 2012 American Control Conference (ACC), Montréal, QC, Canada, 27 June 2012; pp. 5169-5173.

23. Zhang, X.F.; Chen, Y.Q. D-stability Based LMI Criteria of Stability and Stabilization for Fractional Order Systems. In Proceedings of the International Design Engineering Technical Conferences and Computers and Information in Engineering Conference, Boston, MA, USA, 2-5 August 2015; pp. 1-6.

24. Carlos, R.; Karina, A.B.; Daniel, C. Robust Filtering for Discrete-Time Linear Parameter-Varying Descriptor Systems. Symmetry 2020, 12, 1871.

25. Gonzalez, A.; Estrada-Manzo, V.; Guerra, T.M. Gain-scheduled $H_{\infty}$ admissibilisation of LPV discrete-time systems with LPV singular descriptor. Int. J. Syst. Sci. 2017, 48, 3215-3224. [CrossRef]

26. Jiao, Z.; Zhong, Y.S. Robust Stability for Fractional-Order Systems with Structured and Unstructured Uncertainties. Comput. Math. Appl. 2012, 64, 3258-3266. [CrossRef]

27. Chen, L.P.; Wu, R.C.; He, Y.G.; Yin, L.S. Robust Stability and stabilization of Fractional-Order Linear Systems with Polytopic Uncertainties Appl. Math. Comput. 2015, 257, 274-284.

28. Zhang, X.F.; Chen, Y.Q. Admissibility and Robust Stabilization of Continuous Linear Singular Fractional Order Systems with The Fractional Order $\alpha$ : The $0<\alpha<1$ Case. ISA Trans. 2018, 82, 42-50.

29. Israel, A.B. On Error Bounds for Generalized Inverses. SIAM J. Numer. Anal. 1966, 3, 585-592. [CrossRef]

30. Xu, S.J.; Darouach, M. On The Robustness of Linear Systems with Nonlinear Uncertain Parameters. Automatica 1998, 34, 1005-1008. [CrossRef] 
31. Li, X.; Desouzat, C. Criteria for Robust Stability And Stabilization of Uncertain Linear Systems with State Delay. Automatica 1997, 33, 1657-1662. [CrossRef]

32. Boyd, S.; Ghaoui, L.; Feron, E.; Balakrishnan, V. Linear Matrix Inequalities in System and Control Theory; Society for Industrial and Applied Mathematics: Philadelphia, PA, USA, 1994.

33. Kaczorek, T. Descriptor Fractional Linear Systems with Regular Pencils. Asian J. Control 2013, 15, 1051-1064. [CrossRef]

Publisher's Note: MDPI stays neutral with regard to jurisdictional claims in published maps and institutional affiliations.

(c) 2020 by the authors. Licensee MDPI, Basel, Switzerland. This article is an open access article distributed under the terms and conditions of the Creative Commons Attribution (CC BY) license (http://creativecommons.org/licenses/by/4.0/). 